\title{
Analysis of the correlative factors in the selection of interbody fusion cage height in transforaminal lumbar interbody fusion
}

\author{
Hongli Wang ${ }^{\dagger}$, Wenjie Chen ${ }^{\dagger}$, Jianyuan Jiang, Feizhou Lu, Xiaosheng Ma ${ }^{*}$ and Xinlei Xia
}

\begin{abstract}
Background: Selecting an interbody cage with appropriate height is one of the key steps in lumbar interbody fusion, and has an important impact on clinical efficacy. How to choose the appropriate height of the cage becomes one of the core problems of lumbar interbody fusion for spine surgeons. However, studies about objective selection criteria on interbody cage height was rare.
\end{abstract}

Methods: One hundred fifty-seven patients with single segment lumbar degenerative diseases treated by TLIF surgery from January 2011 to July 2013 were retrospectively analyzed. Parameters analyzed included: gender, age, body height, clinical diagnosis, pathological segment location and the intervertebral height of pathological segment, pathological segment activity, the intervertebral height of the adjacent segments. And further to analyze the correlation between these parameters and interbody cage height. By measuring the intervertebral height of pathological segment and normal segment to calculate the regression equation of interbody cage height.

Results: The average interbody cage height of male patients $(12.38 \pm 1.43) \mathrm{mm}$ was significantly higher than female $(11.62 \pm 1.45) \mathrm{mm}(p<0.001)$. The L4-5 segment interbody cage height $(12.11 \pm 1.38) \mathrm{mm}$ was significantly greater than the L5-S1 $(11.25 \pm 1.32) \mathrm{mm}(p=0.04)$. Body height, the intervertebral height of pathological segment, and the middle intervertebral heigh of upper adjacent segment were highly positively correlated to the interbody cage height. The range of interbody cage height used in transforaminal lumbar interbody fusion for Chinese patients with lumbar degenerative diseases was: L3-4 (11.28 \pm 3.29$) \mathrm{mm} \sim(12.76 \pm 2.40) \mathrm{mm}, \mathrm{L} 4-5$ (11.62 \pm 2.89$)$ $\mathrm{mm} \sim(13.18 \pm 1.91) \mathrm{mm}, \mathrm{L} 5-\mathrm{S} 1(10.52 \pm 2.22) \mathrm{mm} \sim(11.90 \pm 2.80) \mathrm{mm}$. The regression equation of interbody cage height was: interbody cage height $=11.123-0.563 *$ (gender) $+0.149 *$ (the middle intervertebral height of pathological segment).

Conclusions: The selection of interbody cage height was influenced by sex, body height, pathological segment location, the intervertebral height of pathological segment and other factors. The interbody cage height for the lower lumbar spine mostly selected 11,12,13 mm, L3-4, L4-5 segment highly selective in general should not be less than $10 \mathrm{~mm}$, and L5-S1 segments height was relatively small, usually not more than $13 \mathrm{~mm}$. The interbody cage height might be selected based on the regression equation of interbody cage height. But, the regression equation maybe need to be verified in a prospective study.

Keywords: Lumbar spine, Lumbar degenerative disease, Lumbar fusion, Interbody fusion, Interbody cage, Radiologic measurement

\footnotetext{
* Correspondence: fudanspine@163.com

${ }^{\dagger}$ Equal contributors

Department of Orthopaedics, Huashan Hospital, Fudan University, Shanghai

200040, China
} 


\section{Background}

Lumbar fusion has been proved excellent clinical effect after more than 100-year development since Albee FH [1] and Hibbs RA [2] first used in 1911, and has become one of the most commonly used operative methods for treating lumbar degenerative diseases which includes posterolateral fusion and interbody fusions [3-5]. The reconstruction of the sagittal balance has become the important purpose of lumbar fusion surgery [6]. Compared with posterolateral fusion, lumbar interbody fusion could restore the supporting role of the anterior column and intervertebral disc height effectively, and provide immediate postoperative stability, which lead to a gradual increase in the proportion of clinical application in recent years [7-10].

Transforaminal lumbar interbody fusion (TLIF) has been widely performed in recent years [11-17], and could effectively restore the intervertebral height, correct lordosis in the pathological lumbar segment and achieve a satisfactory prognosis $[8,9,18,19]$. The lumbar fusion cage, which has been generally accepted, is an alternative method to autologous bone graft, and is mainly used to fill the gap in height after the discectomy and the removal of the superior and inferior cartilage endplates in the diseased segment. Meanwhile, the lordosis in the diseased segment is corrected through the strong fixation using the screw-rod system in the maintenance of base height via the fusion cage. Hsieh PC et al. [6] reported that the local disc angle became smaller after TLIF, which was considered to be caused by an inappropriate posterior position of the implanted fusion cage. This result indicated that the height and position of the implanted fusion cage pathological the recovery from lumbar lordosis and the postoperative prognosis.

Selecting an interbody cage with appropriate height is one of the key steps in lumbar interbody fusion, and has an important impact on clinical efficacy. An oversized cage will not only lead to over distraction of intervertebral space, which may increase abnormal stress in the adjacent segments resulting in increasing the incidence of degeneration of the adjacent segments, but will increase the chance to injury the nerve roots. Using a fusion cage too low in height, on the other hand, will fail to restore the intervertebral height and lordosis, and may lead to severe complications such as cage migration and fusion failure $[20,21]$. Thus, how to choose the appropriate height of the cage becomes one of the core problems of lumbar interbody fusion for spine surgeons. But the interbody cage height in TLIF has long been determined by surgeons mostly based on their operational experience. Hence, it is meaningful to investigate the related factors in the selection of interbody cage height.

The present study attempt to compare the differences in the height of fusion cages used for different disease types or different affected segments based on body height, age, diagnosis, pathological segment location and imaging data collected from patients treated with TLIF. The aim of this study was to measure the intervertebral disc height of normal and pathological segments affected by degenerative diseases, to investigate the factors related to the selection of fusion cage height and to provide reference for height selection of cages in TLIF for patients with lumbar degenerative diseases. This study was conducted with approval from the Ethics Committee of Huashan Hospital, Fudan University. Written informed consent was obtained from all participants before the operation.

\section{Methods}

\section{Patient selection}

We retrospectively analyzed 157 consecutive patients who underwent TLIF for one-segment lumbar degenerative diseases between January 2011 and July 2013 based on following inclusion criteria: 1 ). The patients were diagnosed as lumbar degenerative diseases (lumbar disc herniation, lumbar spinal stenosis or lumbar degenerative spondylolisthesis) in L3-4, L4-5 or L5-S1; 2). The patients underwent single-level TLIF with one interbody cage implanted; 3 ). During the follow-up, there were no complications such as fusion failure, cage retropulsion or pseudarthrosis formation in at least 6 months; 4). The upper and lower adjacent lumbar intervertebral discs were Pfirrmann grade $\leq$ III [22]. This study was conducted with approval from the Ethics Committee of Huashan Hospital, Fudan University. Written informed consent was obtained from all patients.

The exclusion criteria were as follows: 1 ). The patients were diagnosed as isthmic spondylolisthesis, lumbar scoliosis, multiple lumbar degenerative diseases, lumbar secondary surgery, one-segment lumbar degenerative diseases in L1-2, L2-3, or one-segment lumbar degenerative diseases with lumbar fractures, severe osteoporosis, spinal canal tumor or other space-occupying lesions. 2). The patients who had two cages implanted. 3). The patients lost of follow-up, or less than 6 months, or had complications such as fusion failure, cage retropulsion or pseudarthrosis formation. 4). There were adjacent lumbar intervertebral discs observed between Pfirrmann Grade IV and Grade V [22].

In the selected patients, there were 68 male patients and 89 female patients, with an average age of $58.0 \pm 12.5$ years and an average body height of $163.5 \pm 8.5 \mathrm{~cm}$. There were 64 patients with lumbar disc herniation, 51 patients with lumbar spinal stenosis and 42 patients with spondylolisthesis. The surgical procedures were performed on L3-4 in 9 patients, L4-5 in 125 patients and L5-S1 in 23 patients (Table 1).

\section{Study design}

All enrolled subjects underwent lumbar anteroposterior, lateral and dynamic radiographs. All imaging parameters 
Table 1 The basic data of 157 patients with one-segment lumbar degenerative diseases

\begin{tabular}{|c|c|c|c|c|c|c|c|c|}
\hline Disc level & Disease type & Male & Female & Average age (y) & Average height (cm) & $\mathrm{LDH}$ & LSS & DLS \\
\hline L3-4 & 9 & 3 & 6 & $60.4 \pm 10.1$ & $164.1 \pm 9.4$ & 3 & 3 & 3 \\
\hline$\llcorner 4-5$ & 125 & 54 & 71 & $59.9 \pm 11.8$ & $163.0 \pm 7.9$ & 48 & 45 & 32 \\
\hline L5-S1 & 23 & 11 & 12 & $48.7 \pm 13.3$ & $166.4 \pm 7.9$ & 23 & 3 & 7 \\
\hline Total & 157 & 68 & 89 & $58.0 \pm 12.5$ & $163.5 \pm 8.5$ & 64 & 51 & 42 \\
\hline
\end{tabular}

$\mathrm{LDH}$ : lumbar disc herniation, LSS: lumbar spinal stenosis, DLS: degenerative lumbar spondylolisthesis

on the X-ray radiographs were measured by two experienced radiologists using the Centricity Enterprise Web v3.0 (General Electric Com., New York). The following specific measurement parameters were included. 1). The measurement of the intervertebral height taken from the lateral position: One straight line was extended tangent to the inferior endplate of the pathological segment. Three lines were then extended from the anterior, posterior edge and midpoint of the superior endplate vertically toward the line from the inferior endplate, and the length of these vertical line segments were defined as the anterior, posterior intervertebral height (AIVH \& $\mathrm{PIVH})$ and the intervertebral height at the midpoint of the pathological segment (MIVH). The same method as described above was used to measure the intervertebral height at the midpoint of the superior and inferior segment, short for s-MIVH and i-MIVH, respectively (Fig. 1a). 2). The range of motion (ROM) of the pathological segment was measured as the difference between the two angles from the radiographs taken at the anterior flexion and posterior extension positions based on the Cobb method (Fig. 1b and c).

\section{Surgical management}

The same group experienced spine surgeons performed all TLIF surgeries operated on each patient using bilateral internal fixation [23]. With adequate intraoperative decompression, a Capstone lumbar interbody fusion cage (Medtronic Sofamor Danek USA Inc., Memphis, Tennessee) was implanted after distraction. The selection criteria of interbody cage height for this group patients as followed: 1) The intervertebral segment had good stability with certain pull-out resistance after implanting the interbody cage. 2) The height and lordosis of operational segment got a good recovery confirmed by intraoperative fluoroscopy. 3) Both the upper and lower bony endplates of operational segment were kept intact,and the lower nerve root was no significant longitudinal traction. Bone fusion in the pathological segment was detected in postoperative follow-up. Among the enrolled subjects, 2 cages at $8 \mathrm{~mm}, 36$ cages at $10 \mathrm{~mm}, 83$ cages at $12 \mathrm{~mm}$ and 36 cages at $14 \mathrm{~mm}$ were implanted.

\section{Data analysis}

SPSS 19.0 statistical software (SPSS Inc., Chicago, Illinois) was used for the statistical analysis. The difference was statistically significant if $p<0.05$. Kruskal-Wallis $\mathrm{H}$ test was used to compare the differences between the diseases, Wilcoxon test was used to compare the differences between different segments, $t$ test was used to compare the differences between MIVH, s-MIVH and s-MIVH, and genders. Associations between the radiological parameters
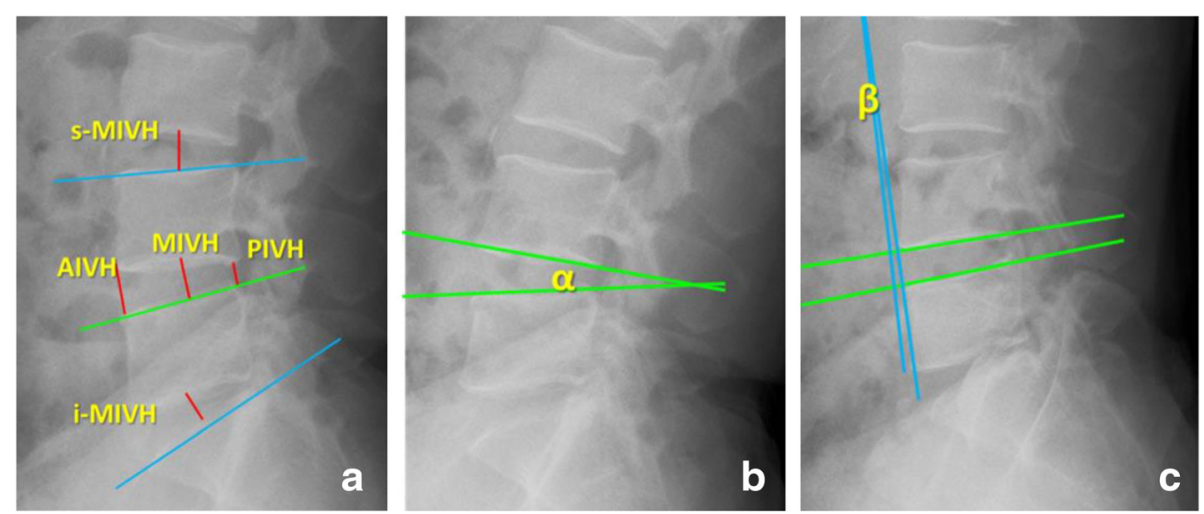

Fig. 1 Measurement methods of the intervertebral height and range of motion. a Measurement methods of the anterior, posterior intervertebral height (AIVH \& PIVH) and the intervertebral height at the midpoint of the pathological segment (MIVH), and the intervertebral height at the midpoint of the superior and inferior segment (s-MIVH and i-MIVH, respectively). $\mathbf{b}$ and $\mathbf{c}$ The range of motion (ROM) of the pathological segment is measured based on the Cobb method, and is the difference between Angle $a$ and $\beta$ 
and the interbody cage height were analyzed with Spearman correlation analysis.

\section{Results \\ Differences in the fusion cages used for different disease types}

The Kruskal-Wallis H Test showed no differences in the height of the fusion cages used for different disease types $(p=0.20)$. For each disease type, no significant difference was observed in pairwise inter-group comparison $(8 \mathrm{~mm}$ fusion cages were not included in the analysis due to the small sample size). The average fusion cage height used was $12.16 \pm 1.30 \mathrm{~mm}$ in the lumbar disc herniation group, $12.00 \pm 1.55 \mathrm{~mm}$ in the lumbar spinal stenosis group and $11.57 \pm 1.43 \mathrm{~mm}$ in the lumbar degeneration spondylolisthesis group.

\section{Differences in fusion cage usage for different segments} Due to the small size of the L3-4 group and the $8 \mathrm{~mm}$ fusion cage group, fusion cage usage was only compared between the L4-5 group and the L5-S1 group using the Wilcoxon rank sum test. A p value of 0.04 was obtained, indicating that the average height of the fusion cages used for diseased L4-5 segment $(12.11 \pm 1.38 \mathrm{~mm})$ was greater than that for the L5-S1 segment $(11.25 \pm 1.32 \mathrm{~mm})$.

\section{Differences in fusion cages used between different genders}

Significant differences were observed in the average height of the fusion cages used between male and female subjects $(p<0.001$; the $8 \mathrm{~mm}$ fusion cage was not included). The average height of the fusion cages used in male subjects $(12.38 \pm 1.43 \mathrm{~mm})$ was greater than that used in female subjects $(11.62 \pm 1.45 \mathrm{~mm})$. (Table 2$)$

\section{Correlation between fusion cage height and index parameters}

A Spearman correlation analysis was performed between the fusion cage height and age, body height, AIVH, MIVH, PIVH, i-MIVH, s-MIVH and ROM of the pathological segment. The fusion cage height showed positive correlations with AIVH, MIVH, PIVH, s-MIVH and body height, with the correlation coefficients in ascending order. With increases in these parameters, the height of the fusion cage increased correspondingly (Table 3).

\section{Intervertebral disc height of normal and pathological segments}

In the present study, the intervertebral height of 291 segments assessed between Pfirrmann Grade I to Grade III were measured. According to Pfirrmann classification of disc degeneration, the intervertebral disc height of Grade I to Grade III indicate no height loss or slightly decrease [22]. So the adjacent segments could be considered as normal or similar to normal intervertebral disc height. The average MIVH of L2-3 to L5-S1 was $12.15 \pm 2.42 \mathrm{~mm}, 12.76 \pm 2.40 \mathrm{~mm}, 13.18 \pm 1.91 \mathrm{~mm}$, $11.90 \pm 2.80 \mathrm{~mm}$, respectively (Table 4 ).

\section{Stepwise regression of fusion cage height}

Stepwise regression equations were obtained using the fusion cage height as the dependent variable and the above parameters as the independent variables, which showed significant differences. These variables included gender (male $=1$, female $=2$ ), AIVH, MIVH, PIVH, s-MIVH and body height. The stepwise regression equation was significant $(p<0.001)$ with the retained two independent variables, gender and MIVH. The equation was as follows: interbody cage height $=11.123-0.563 *$ gender + $0.149 * \mathrm{MIVH}$.

\section{Discussion}

Unlike the prone position the subjects assumed in the fusion surgery, the basic function of the human spine is load-bearing, and thus, the height and curvature formed with the fusion cage implanted in a non-load-bearing state are likely to deviate from those under the normal upright position. Literature [24] reports indicated that the normal human lumbar exhibited significantly increased local disc angle in the L2-3 and L3-4 segments and markedly reduced local disc angle in the L5-S1 segment in response to axial stress. This result suggested

Table 2 Statistical data of the selection of fusion cage height with different diagnosis, segments and gender

\begin{tabular}{lllllll}
\hline Fusion cage height & $8 \mathrm{~mm}$ & $10 \mathrm{~mm}$ & $12 \mathrm{~mm}$ & $14 \mathrm{~mm}$ & Average height $(\mathrm{mm})$ & $p$ \\
\hline LDH & 0 & 11 & 37 & 16 & $12.16 \pm 1.30$ & $12.00 \pm 1.55$ \\
LSS & 1 & 12 & 24 & 14 & $11.57 \pm 1.43$ \\
DLS & 1 & 13 & 22 & 6 & $12.22 \pm 1.86$ \\
L3-4 & 0 & 3 & 2 & 4 & $12.11 \pm 1.38$ \\
L4-5 & 1 & 24 & 69 & 1 & $11.25 \pm 1.32$ \\
L5-S1 & 1 & 9 & 37 & 23 & $12.38 \pm 1.43$ \\
Male & 2 & 30 & 46 & 13 & $11.62 \pm 1.45$ \\
Female & 0 & 30 & 12 & $<0.001$
\end{tabular}

LDH: lumbar disc herniation, LSS: lumbar spinal stenosis, DLS: degenerative lumbar spondylolisthesis 
Table 3 Correlation between fusion cage height and index parameters

\begin{tabular}{lllllllll}
\hline & Age & Body height & AIVH & MIVH & PIVH & s-MIVH & i-MIVH & ROM \\
\hline Coefficient of correlation & - & 0.169 & 0.331 & 0.331 & 0.314 & 0.183 & - & - \\
$p$ & 0.850 & 0.034 & $<0.001$ & $<0.001$ & $<0.001$ & 0.022 & 0.143 & 0.187 \\
\hline
\end{tabular}

AIVH: anterior posterior intervertebral height, MIVH: intervertebral height at the midpoint, $\mathrm{PIVH}$ : posterior intervertebral height, s-MIVH: intervertebral height at the midpoint of the superior segment, i-MIVH: intervertebral height at the midpoint of the inferior segment, ROM: range of motion

that in response to axial stress, the upper lumbar spine tends to disperse the force toward the medial and posterior column, while the lower lumbar spine compresses the intervertebral height to some extent. The differences in the lumbar intervertebral height are determined by the anatomical structure and the physiological characteristics of the lumbar spine itself, which is consistent with the finding from the present study that the height of the fusion cage used in the surgery correlated with the segment pathological by the disease rather than with the disease type itself. On the other hand, Videman $\mathrm{T}$ et al. [25] found that the lower lumbar intervertebral height decreased with age in an elderly sample. Meanwhile, large individual variance in the height reduction was found in the same study, which might explain why age was not found to correlate with the height of the fusion cage in the present study.

According to the results from this study, the MIVH of the pathological segments from L3-4 to L5-S1 was $11.28 \pm 3.29 \mathrm{~mm}, 11.62 \pm 2.89 \mathrm{~mm}, 10.52 \pm 2.22 \mathrm{~mm}$, which was significantly lower than those of the normal segments: $12.76 \pm 2.40 \mathrm{~mm}, 13.18 \pm 1.91 \mathrm{~mm}$ and $11.90 \pm 2.80 \mathrm{~mm}$, respectively. Therefore, the proper cage height of Chinese population should be within the range of the two sets of data mentioned above of each segment. The average cage height of each segment we actually used was $12.22 \pm 1.86 \mathrm{~mm}, 12.11 \pm 1.38$ and $11.25 \pm 1.32 \mathrm{~mm}$, which, however, was exactly within the proper range that proved the adequacy of the choice of the cage height in this sample. Combined with common cage scale, we recommend that the cage height of L3-4 and L4-5 level via TLIF should be 11,12 or $13 \mathrm{~mm}$, while for L5-S1 level, 10, 11 or $12 \mathrm{~mm}$ would be more appropriate, which means, in Chinese population, the interbody cage height for patients with lumbar degenerative diseases should not be lower than $10 \mathrm{~mm}$ in L3-4 and L4-5 segment, and not be greater than $13 \mathrm{~mm}$ in L5-S1 segment. In addition, the stepwise regression equation: interbody cage height $=11.123-0.563 *$ gender $+0.149 * \mathrm{MIVH}$, presented in the study could be used to estimate the interbody cage height preliminarily during the operation.

There were some limitations in this retrospective study. The small sample size and the large deviation of segmental distribution might have an impact on the accuracy of some parameters, especially for L3-4 segment. The results of L3-4 segment need to be more careful handling. Besides, the selection of the size of the interbody cage for each patient in this sample group was mainly depend on the surgeons' experience during the operation, of which the correctness should be proved in further studies, despite of the height of the cages used was within the range of the intervertebral height at the midpoint of the pathological and normal segments.

\section{Conclusion}

In summary, the selection of interbody cage height was influenced by gender, body height, pathological segment location, the intervertebral height of pathological segment and other factors. The interbody cage height for the lower lumbar spine mostly selected was 11,12,13 mm, L3-4, L4-5 segment highly selective in general should not be less than $10 \mathrm{~mm}$, and L5-S1 segments height was relatively small, usually not more than $13 \mathrm{~mm}$. The interbody cage height might be calculated before surgery for reference based on the regression equation as follows: interbody cage height $=$ $11.123-0.563 *$ gender $+0.149 * \mathrm{MIVH}$. But, the regression equation maybe need to be verified in a prospective study.

Table 4 Intervertebral disc height of normal and pathological segment

\begin{tabular}{|c|c|c|c|c|c|}
\hline \multirow{2}{*}{$\begin{array}{l}\text { Parameters } \\
\text { Segments }\end{array}$} & \multicolumn{2}{|l|}{ Number of segments } & \multicolumn{2}{|l|}{ MIVH } & \multirow{2}{*}{$\begin{array}{l}\text { Height of } \\
\text { fusion cage }\end{array}$} \\
\hline & Pathological segments & Normal segments & Pathological segments & Normal segments & \\
\hline L3-4 & 9 & 125 & $11.28 \pm 3.29$ & $12.76 \pm 2.40$ & $12.22 \pm 1.86$ \\
\hline$\llcorner 4-5$ & 125 & 32 & $11.62 \pm 2.89$ & $13.18 \pm 1.91$ & $12.11 \pm 1.38$ \\
\hline L5-S1 & 23 & 125 & $10.52 \pm 2.22$ & $11.90 \pm 2.80$ & $11.25 \pm 1.32$ \\
\hline
\end{tabular}




\section{Abbreviations}

TLIF: Transforaminal lumbar interbody fusion; LDH: Lumbar disc herniation; LSS: Lumbar spinal stenosis; DLS: Degenerative lumbar spondylolisthesis; AIVH: The anterior intervertebral height; PIVH: The posterior intervertebral height; MIVH: The intervertebral height at the midpoint of the pathological segment; s-MIVH: The intervertebral height at the midpoint of the superior segment; i-MIVH: The intervertebral height at the midpoint of the inferior segment; ROM: The range of motion.

\section{Competing interests}

The authors declare that they have no competing interests.

\section{Authors' contributions}

Author contributions to the study and manuscript preparation include the following. MXS carried out the design of the study. WHL carried out the acquisition of data, and drafted the manuscript. CWJ also carried out the acquisition of data, and revised the manuscript. XXL performed the statistical analysis. JJY participated in the design of this study and coordination and helped to draft the manuscript. LFZ also participated in its design and coordination and helped to draft the manuscript. All authors read and approved the final manuscript.

\section{Acknowledgements}

We thank Lixun Wang,MD who provided statistical analysis suggestions, and sincerely thank all the patients for their participation in this study. The work was supported by the 2012 Key Medical Projects of Shanghai Committee of Science and Technology (No. 12411951201) and 2014 General Program of National Natural Science Foundation of China (No. 81472036).

Received: 2 August 2015 Accepted: 5 January 2016

Published online: 12 January 2016

\section{References}

1. Albee FH. Transplantation of a portion of the tibia into the spine for Pott's disease: a preliminary report 1911. Clin Orthop Relat Res. 2007:460:14-6.

2. Hibbs RA. An operation for progressive spinal deformities: a preliminary report of three cases from the service of the orthopaedic hospital. 1911. Clin Orthop Relat Res. 2007:460:17-20.

3. Groff MW, Dailey AT, Ghogawala Z, Resnick DK, Watters 3rd WC, Mummaneni PV, et al. Guideline update for the performance of fusion procedures for degenerative disease of the lumbar spine. Part 12: pedicle screw fixation as an adjunct to posterolateral fusion. J Neurosurg Spine. 2014;21:75-8.

4. Mummaneni PV, Dhall SS, Eck JC, Groff MW, Ghogawala Z, Watters 3rd WC, et al. Guideline update for the performance of fusion procedures for degenerative disease of the lumbar spine. Part 11: interbody techniques for lumbar fusion. J Neurosurg Spine. 2014;21:67-74.

5. Noshchenko A, Hoffecker L, Lindley EM, Burger EL, Cain CM, Patel W. Perioperative and longterm clinical outcomes for bone morphogenetic protein versus iliac crest bone graft for lumbar fusion in degenerative disk disease: systematic review with meta-analysis. J Spinal Disord Tech. 2014 27(3):117-35.

6. Hsieh PC, Koski TR, O'Shaughnessy BA, Sugrue P, Salehi S, Ondra S, et al. Anterior lumbar interbody fusion in comparison with transforaminal lumbar interbody fusion: implications for the restoration of foraminal height, local disc angle, lumbar lordosis, and sagittal balance. J Neurosurg Spine. 2007;7:379-86.

7. Pannell WC, Savin DD, Scott TP, Wang JC, Daubs MD. Trends in the surgical treatment of lumbar spine disease in the United States. Spine J. 2015;15(8): 1719-27.

8. Watkins RG, Hanna R, Chang D, Watkins 3rd RG. Sagittal alignment after lumbar interbody fusion: comparing anterior, lateral, and transforaminal approaches. J Spinal Disord Tech. 2013;27:253-6.

9. Yson SC, Santos ER, Sembrano JN, Polly Jr DW. Segmental lumbar sagittal correction after bilateral transforaminal lumbar interbody fusion. J Neurosurg Spine. 2012;17:37-42. Epub 2012 May 11

10. Zhou J, Wang B, Dong J, Li X, Zhou X, Fang T. Lin H (2011) Instrumented transforaminal lumbar interbody fusion with single cage for the treatment of degenerative lumbar disease. Arch Orthop Trauma Surg. 2011;131(9):1239-45.

11. Harms J, Rolinger $\mathrm{H}$. A one-stager procedure in operative treatment of spondylolistheses: dorsal traction-reposition and anterior fusion (author's transl). Zeitschrift fur Orthopadie und ihre Grenzgebiete. 1982;120:343-7.
12. Harris BM, Hilibrand AS, Savas PE, Pellegrino A, Vaccaro AR, Siegler S, et al. Transforaminal lumbar interbody fusion: the effect of various instrumentation techniques on the flexibility of the lumbar spine. Spine. 2004:29:E65-70.

13. Hoy K, Bunger C, Niederman B, Helmig P, Hansen ES, Li H, et al. Transforaminal lumbar interbody fusion (TLIF) versus posterolateral instrumented fusion (PLF) in degenerative lumbar disorders: a randomized clinical trial with 2-year follow-up. Eur Spine J. 2013;22:2022-9. Epub 2013 Apr 13.

14. Lauber S, Schulte TL, Liljenqvist U, Halm H, Hackenberg L. Clinical and radiologic 2-4-year results of transforaminal lumbar interbody fusion in degenerative and isthmic spondylolisthesis grades 1 and 2. Spine. 2006:31:1693-8.

15. Pumberger M, Hughes AP, Girardi FP, Gogia J, Kotwal SY, Thaler C, et al Influence of surgical experience on the efficiency of discectomy in TLIF: a cadaveric testing in 40 levels. J Spinal Disord Tech. 2012;25:E254-258.

16. Rosenberg WS, Mummaneni PV. Transforaminal lumbar interbody fusion: technique, complications, and early results. Neurosurgery. 2001;48:569-74. discussion 574-565.

17. Zhang Q, Yuan Z, Zhou M, Liu H, Xu Y, Ren Y. A comparison of posterior lumbar interbody fusion and transforaminal lumbar interbody fusion: a literature review and meta-analysis. BMC Musculoskelet Disord. 2014;15:367.

18. Ould-Slimane M, Lenoir T, Dauzac C, Rillardon L, Hoffmann E, Guigui P, et al. Influence of transforaminal lumbar interbody fusion procedures on spinal and pelvic parameters of sagittal balance. Eur Spine J. 2011;21:1200-6. Epub 2011 Dec 17.

19. Xiao Y, Li F, Chen Q. Transforaminal lumbar interbody fusion with one cage and excised local bone. Arch Orthop Trauma Surg. 2009:130:591-7. Epub 2009 Jun 30

20. Aoki Y, Yamagata M, Nakajima F, Ikeda Y, Shimizu K, Yoshihara M, et al. Examining risk factors for posterior migration of fusion cages following transforaminal lumbar interbody fusion: a possible limitation of unilateral pedicle screw fixation. J Neurosurg Spine. 2010;13:381-7.

21. Uzi EA, Dabby D, Tolessa E, Finkelstein JA. Early retropulsion of titaniumthreaded cages after posterior lumbar interbody fusion: a report of two cases. Spine. 2001;26:1073-5

22. Pfirrmann CW, Metzdorf A, Zanetti M, Hodler J, Boos N. Magnetic resonance classification of lumbar intervertebral disc degeneration. Spine. 2001;26:1873-8.

23. Jiang JY, Ma X, Lu FZ, Wang HL, Chen WJ, Ma XS, et al. The anatomic study and clinical significance of the modified transforaminal lumbar interbody fusion. Zhonghua wai ke za zhi [Chinese journal of surgery]. 2009:47:1100-3.

24. Hioki A, Miyamoto K, Shimizu K, Inoue N. Test-retest repeatability of lumbar sagittal alignment and disc height measurements with or without axial loading: a computed tomography study. J Spinal Disord Tech. 2011;24:93-8.

25. Videman T, Battie MC, Gibbons LE, Gill K. Aging changes in lumbar discs and vertebrae and their interaction: a 15-year follow-up study. Spine J. 2013;14:469-78. Epub 2013 Nov 19

\section{Submit your next manuscript to BioMed Central and we will help you at every step:}

- We accept pre-submission inquiries

- Our selector tool helps you to find the most relevant journal

- We provide round the clock customer support

- Convenient online submission

- Thorough peer review

- Inclusion in PubMed and all major indexing services

- Maximum visibility for your research

Submit your manuscript at www.biomedcentral.com/submit 